\title{
ABUNDANCES IN HALO POPULATION STARS
}

\author{
CHRISTOPHER SNEDEN \\ Department of Astronomy and McDonald Observatory \\ University of Texas, Austin, TX 78712
}

\section{Introduction}

The quest to define early galactic nucleosynthesis has drawn on the efforts of many observers and theoreticians over the past few decades. Significant insights on galactic halo chemical evolution have been revealed through the use of high resolution, high $\mathrm{S} / \mathrm{N}$ spectroscopy to determine abundances in stars with metallicities ranging over three orders of magnitude. In the process, many galactic nucleosynthesis ideas that were firmly established only a decade ago now are either disproved, or are currently under challenge, or are weighed down with multiple known exceptions. The galactic halo has a rich chemical history, and we are just beginning to understand just how much work still remains before we can decipher its evolution.

In this brief review we will discuss some recent halo abundance studies; for general reviews the reader is referred to, eg., Cayrel (1996) or McWilliam (1997). Our discussion will necessarily be limited. For example, we will not consider abundance trends in LiBeB group, as those elements are explored by other contributors to this volume. The growing literature on light element abundance variations within globular clusters must be left to other reviews. Instead, in the next four sections we discuss in turn the questions of: a) anomalies in $\alpha$-capture abundance ratios; b) overall trends in "light" and Fe-peak elements in ultra-metal-poor stars; c) relative r- and sprocess contributions to neutron-capture elemental abundances as functions of metallicity; and d) new indicators of the very heavy element abundance distribution in the early galaxy.

\section{Anomalous $\alpha$-Capture Abundance Ratios}

Pioneering early studies (eg., Wallerstein et al. 1962) showed that light even-Z elements $\mathrm{O}, \mathrm{Mg}, \mathrm{Si}, \mathrm{Ca}$ and $\mathrm{Ti}$ are overabundant in metal-poor halo 
stars: [element $/ \mathrm{Fe}] \simeq+0.3$ to +0.5 . These elements, generated in $\alpha$-capture fusion reactions, are efficiently produced in high-mass stars and returned to the ISM in Type II supernovae. Therefore the $\alpha$-element overabundances in metal-poor stars imply that short-lived high mass stars dominated early galactic halo nucleosynthesis. It has also been asserted that the $[\alpha / \mathrm{Fe}]$ ratios of all stars with $[\mathrm{Fe} / \mathrm{H}]$ metallicities $<-1$ are nearly identical, thus allegedly proving that the IMF was the same in different parts of the halo or that the halo ISM gas was able to mix on time scales short compared to the formation of subsequent stellar generations.

However, not all halo stars have elevated $\alpha$-capture abundances; some even have $[\alpha / \mathrm{Fe}]<0$. Brown et al. (1997) found that red giants in two outer halo globular clusters (Rup 106 and Pal 12) have metallicities $[\mathrm{Fe} / \mathrm{H}] \simeq$ -1.4 and -0.9 but weak $\alpha$ element abundances: $\langle[\alpha / \mathrm{Fe}]\rangle \sim 0$. Carney et al. (1997) report that the halo field star $\mathrm{BD}+80^{\circ} 245$ has $[\mathrm{Fe} / \mathrm{H}] \simeq-$ 1.85 but $\langle[\alpha / \mathrm{Fe}]>\sim-0.25$. There is at least one metal-poor star with an anomalously low $[\mathrm{Mg} / \mathrm{Fe}]$ ratio in the sample of Fuhrmann et al. (1995). Finally, Nissen \& Schuster (1997) have completed a careful abundance study of 30 stars in the metallicity range $-0.4>[\mathrm{Fe} / \mathrm{H}]>-1.3$; about a quarter of their sample have substantially lower $\alpha$-element abundances than the rest.

A common characteristic of the low- $\alpha$ stars in these studies is that they have orbits that take them to the outer halo regions of the galaxy. Clearly a new halo sub-population is emerging. The fraction of low- $\alpha$ stars cannot be assessed at the present time, but one can at least speculate on their nucleosynthetic origin. The papers cited above suggest the heavy elements in these stars were enriched in the ejecta of Type Ia supernovae, which are much more efficient producers of iron than the $\alpha$-elements. Type Ia $\mathrm{SNe}$ arise from long-lived stars, and their influence on overall trends in abundance ratios are not apparent until $[\mathrm{Fe} / \mathrm{H}]>-1$, when their presence is signaled by declining $[\alpha / \mathrm{Fe}]$ ratios. Therefore, Nissen $\&$ Schuster suggest that the low- $\alpha$ stars may have formed in ISM regions of abnormally low density, and thus relatively low star-formation rate. This would retard the chemical enrichment of the gas by high-mass Type II SNe, allowing the "delayed" iron production from the Type Ia SNe to occur at relatively low metallicities. Such lower density ISM environments may have been regions of our own galactic halo, or perhaps these stars were produced in nearby dwarf galaxies and later captured by our galaxy; the stellar statistics are insufficient to decide at the present time.

A final clue comes from the depressed levels of the odd- $Z$ light elements sodium and possibly aluminum in the low- $\alpha$ stars. Apparently all such stars discovered to date have $[\mathrm{Na} / \mathrm{Fe}] \leq-0.3$, and this is another indication that Type Ia nucleosynthesis (poor in Na production) was at work. Nissen \& Schuster (1997) suggest that searches for these anomalies be made in 
other halo star samples. Such studies must be done with carefully-selected control samples of normal halo stars, but a small test can be performed here. Pilachowski et al. (1996) reported light element abundances for nearly 60 field giants (to compare with the abundances of globular cluster giants). From their sample, we list in Table 1 some mean values of $[\mathrm{Na} / \mathrm{Fe}]$ in five domains of $[\alpha / \mathrm{Fe}]$, where $\alpha$ for a star here is defined as the mean of the $\mathrm{Mg}, \mathrm{Si}$, and $\mathrm{Ca}$ abundances for that star.

TABLE 1. Field Giants: Mean Abundances

\begin{tabular}{ccc}
\hline$[\alpha / \mathrm{Fe}]$ range & $\langle[\mathrm{Na} / \mathrm{Fe}]\rangle$ & No. Stars \\
\hline+0.05 to +0.14 & $-0.34 \pm 0.07$ & 6 \\
+0.15 to +0.24 & $-0.28 \pm 0.04$ & 10 \\
+0.25 to +0.34 & $-0.15 \pm 0.03$ & 23 \\
+0.35 to +0.44 & $-0.14 \pm 0.05$ & 16 \\
+0.45 to +0.54 & $+0.04 \pm 0.10$ & 4 \\
\hline
\end{tabular}

The correlation between the $\alpha$-elements and sodium certainly seems present, but confirmation must await a more detailed study. An advantage of Nissen \& Schuster's study was the concentration on relatively unevolved nearby stars for which good kinematical information is available. That will not be very easy for the Pilachowski et al. (1996) giant stars, most of whom do not have reliable parallaxes even in the post-HIPPARCOS era.

\section{Abundance Trends in Ultra-Metal-Poor Stars}

Ultra-metal-poor (hereafter, UMP) stars are defined here as those with $[\mathrm{Fe} / \mathrm{H}]<-2.5$. This metallicity is the lower limit for globular clusters, and it also is the metallicity at which Fe-peak elements in field stars begin to exhibit severe departures from solar abundance ratios. Much of our knowledge of chemical compositions of UMP stars has come about quite recently, thanks largely to the efforts of the past decade to substantially increase the number of candidate UMP stars. Cayrel (1996) discusses the new UMP surveys at length, but we take special note of the ongoing Beers et al. (1992) survey that has significantly augmented the candidate star sample.

Ryan et al. $(1991,1996)$ and McWilliam et al. (1995) have conducted the most comprehensive abundance surveys of UMP stars; see these papers for references to smaller-sample analyses of UMP stars. The Ryan et al. (1996) paper is especially useful, as it includes abundance results from a 
variety of investigators, thus providing estimates of both intrinsic (scatter) errors within individual studies and external (scale) errors between different studies. Statistical methods are applied to derive robust mean abundance trends, and these are then compared to the galactic chemical evolution models of Timmes et al. (1995). The trustworthiness of observational results are also discussed by Ryan et al.; for example, the compilation of $[\mathrm{Ca} / \mathrm{Fe}]$ values in their Figure 2 shows reasonably small scatter (neglecting the few points that may represent $\alpha$-poor stars; see above) and satisfactory agreement with the Timmes et al. predicted trend. By way of contrast, the observational situation for $\mathrm{Al}$ is still poor, and Ryan et al. give a succinct discussion of problems, which include possible non-LTE effects, clashes between abundances from different Al I transitions employed in studies of high and low metallicity stars, and large offsets between different investigations of the same stars. Their cautions should be heeded; renewed attention should be paid to those elements where observational uncertainties prevent meaningful comparison to theory.

The most noteworthy general result of UMP abundance surveys has been the identification of sharply non-solar abundance ratios among $\mathrm{Fe}-$ peak elements, shown most dramatically in small $[\mathrm{Cr} / \mathrm{Fe}]$ and large $[\mathrm{Co} / \mathrm{Fe}]$ ratios. Sub-solar $[\mathrm{Mn} / \mathrm{Fe}]$ in "ordinary" metal-poor stars $([\mathrm{Fe} / \mathrm{H}]>-2.5)$ had been found previously (eg., Gratton 1989), but the new surveys identify a further decrease in this abundance ratio at UMP metallicities. The Timmes et al. (1995) chemical evolution models predict the trends of [Sc/Fe] and $[\mathrm{Mn} / \mathrm{Fe}]$ with $[\mathrm{Fe} / \mathrm{H}]$, and even the possible increase in observed $[\mathrm{Ni} / \mathrm{Fe}]$ values at $[\mathrm{Fe} / \mathrm{H}]<-3$ is suggested by these models. But the $\mathrm{Co}$ and $\mathrm{Cr}$ variations are much more severe than those suggested by the models, even though there are hints (see Figure 4 of Ryan et al. 1996) that some tinkering with model parameters could produce rough agreement. The simplest explanation for the Fe-peak trends is that Fe-peak nucleosynthesis in lowmetallicity Type II SNe is driven toward the heavier elements $(\mathrm{Co}, \mathrm{Ni})$ at the expense of the lighter ones ( $\mathrm{Cr}, \mathrm{Mn}$ ) by invoking an $\alpha$-rich freezeout to the conditions during the explosive Si-burning.

Left in limbo by this pleasing scenario are $\mathrm{Cu}$ and $\mathrm{Zn}$, elements at the high-Z end of the Fe-peak. Extant observations (Sneden et al. 1991) suggest that $[\mathrm{Zn} / \mathrm{Fe}] \simeq 0$ at all metallicities, and that $\mathrm{Cu}$ abundances decline sharply with decreasing metallicity $([\mathrm{Cu} / \mathrm{Fe}] \sim-1$ at $[\mathrm{Fe} / \mathrm{H}] \sim-3)$. Explosive Si-burning is apparently responsible for $\mathrm{Zn}$ production, and thus a puzzle emerges: if $\alpha$-rich freezeout created overabundances of the heaviest Fe-peak elements in UMP stars, why is $[\mathrm{Zn} / \mathrm{Fe}] \simeq 0$ observed at all metallicities? A small overabundance of $\mathrm{Ni}$ should be accompanied by a very large overabundance of $\mathrm{Zn}$. The nucleosynthetic origin of $\mathrm{Cu}$ is not at all clear; it may be synthesized in explosive neon burning or it maybe the lightest of 
the s-process neutron-capture elements. If it could be conclusively shown that $\mathrm{Fe}$-peak element production bypasses $\mathrm{Cu}$, then it might be an effective surrogate for discussions of s-process nucleosynthesis in low metallicity environments. However, the Sneden et al. survey included only one star with $[\mathrm{Fe} / \mathrm{H}]<-2.7$, and the other UMP surveys do not report abundances for either $\mathrm{Cu}$ or $\mathrm{Zn}$. Thus the abundances for these two key elements must be considered simply as unknown in UMP stars, and new observational studies of them should be undertaken.

\section{Neutron-Capture Element Abundances at Low Metallicities}

Nearly two-thirds of the stable elements in the periodic table are synthesized in neutron bombardment fusion chains. The neutron-capture rates compete with $\beta$-decay rates in building almost all nuclei with $\mathrm{Z}>30$. Low neutron fluxes (the so-called s-process) create unstable nuclei that undergo $\beta$ decays between successive neutron captures, and element synthesis proceeds along the valley of $\beta$-stability. On the other hand, high neutron fluxes (the r-process) create extremely heavy isotopes of nuclei (up to the neutrondrip line) that then rapidly decay back toward the valley of $\beta$-stability after the cessation of the neutron flux. The s-process may occur during the quiescent stages of stellar evolution (such as during helium burning), while the most likely site for the r-process is during formation of a neutron star in a Type II supernova detonation (eg. Meyer 1994). Thus in the early galaxy when Type II supernovae dominated the light element synthesis (the $[\alpha / \mathrm{Fe}]$ ratios of most metal-poor stars are large), one expects substantial r-process contributions to the neutron-capture element abundances as well.

Among the heavier neutron-capture elements, $\mathrm{Ba}$ isotopes are synthesized most easily by the s-process, while Eu isotopes are readily made in the r-process. Thus their elemental abundances as functions of metallicity can give some indication of the relative $\mathrm{r} / \mathrm{s}$ contributions in the early galaxy. In Figure 1 we have gathered abundances from several several large-sample studies, including some preliminary results from the new field star abundance survey of Burris et al. (1998). Excluded from Figure 1 are abundances of $\mathrm{CH}$ stars and other stars whose neutron capture abundance patterns clearly indicate that they have been polluted during their lives by s-process material from within or from a close companion. For most stars $[\mathrm{Ba} / \mathrm{Fe}]$ values decline at metallicities $[\mathrm{Fe} / \mathrm{H}]<-2$, while $[\mathrm{Eu} / \mathrm{Fe}]$ values remain large. At $[\mathrm{Fe} / \mathrm{H}] \sim-3,[\mathrm{Ba} / \mathrm{Eu}] \sim-0.8$, which is close to the solar-system $\mathrm{r}$-process fractional abundance ratio of these elements (eg. Käppeler et al. 1989). This point has been made before (see the references in McWilliam 1997); but it is worth noting that all investigators agree on the basic abundance trends. This is very consistent with $\mathrm{r}$-process synthesis from supernovae in the early 


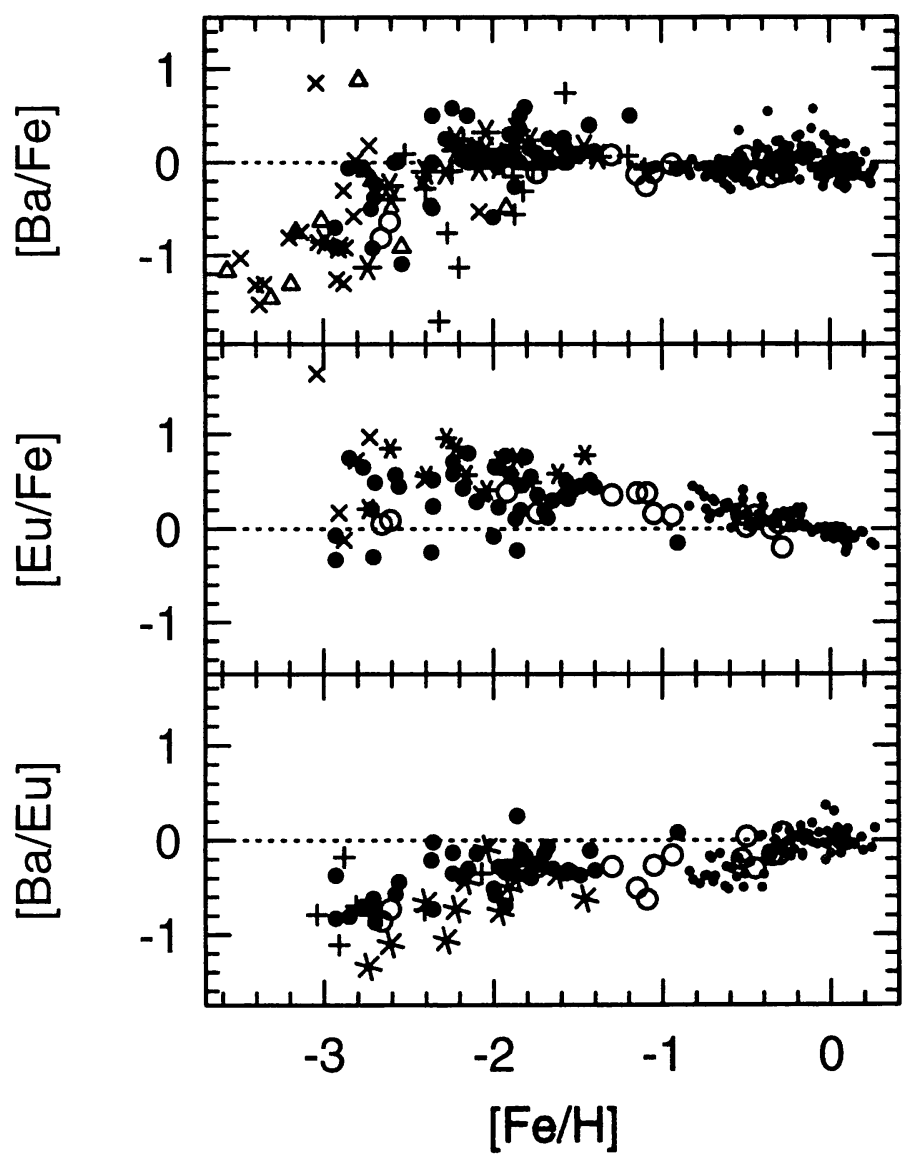

Figure 1. Ba and Eu abundances as functions of metallicity. The horizontal dotted lines represent the solar abundance ratios. The small circles are from Edvardsson et al. (1993) and Woolf et al. (1995), the large circles are the subgiant and giant stars of Burris et al. (1998), the asterisks are from Magain (1989) and Zhao \& Magain (1990), the open triangles are from Ryan et al. (1991,1996), the x's are from McWilliam et al. (1995), the plus signs are from François (1996), and the open circles are from Gratton \& Sneden (1994).

galaxy, without substantial s-process contribution.

Support for the elemental abundance results comes from a new $\mathrm{Ba}$ isotopic abundance study by Gacquer \& François (1998). They discuss their work in this volume, showing that the width of the Ba II $4554 \AA$ resonance line in the UMP subgiant HD 140283 can be matched only through a higher contribution by the $\mathrm{r}$-process to the $\mathrm{Ba}$ abundance in this star than in the solar system. Their analysis clears up the puzzling initial isotopic $\mathrm{Ba}$ estimate for this star (Magain 1995), which suggested a large s-process 
contribution. $\mathrm{Ba}$ isotopic analyses should be extended to stars of a large metallicity range, hopefully to settle this question once and for all.

Although the overall trends of $[\mathrm{Ba} / \mathrm{Fe}]$ and $[\mathrm{Eu} / \mathrm{Fe}]$ with metallicity are clear, these abundance ratios exhibit $\sim 1$ dex of star-to-star scatter at any $[\mathrm{Fe} / \mathrm{H}]<-1$. However, as argued by Gilroy et al. (1988) and extended by Burris et al. (1998), this scatter is largely real, and is not dominated by observational errors. Examples abound of metal-poor stars with nearly identical absorption-line strengths for all spectral features except those of the neutron-capture elements. The star-to-star scatter for $[\mathrm{Ba} / \mathrm{Eu}]$ is substantially smaller than for either $[\mathrm{Ba} / \mathrm{Fe}]$ or $[\mathrm{Eu} / \mathrm{Fe}]$. The $[\mathrm{Ba} / \mathrm{Eu}]$ ratio is an excellent indicator of the steadily increasing significance of r-process nucleosynthesis with decreasing metallicity, but the ratio of either element with respect to Fe clearly shows the non-homogeneity of "bulk" neutron-capture element abundance levels in galactic halo gas.

This inhomogeneity is vividly illustrated by CS 22892-052, a UMP star from the Beers et al. (1992) survey that was included in the McWilliam et al. (1995) abundance study. Preliminary reconnaissance of those spectra showed that CS 22892-052 has very large overabundances of all neutroncapture elements (in Figure 1, this star appears at $[\mathrm{Fe} / \mathrm{H}] \sim-3.1,[\mathrm{Eu} / \mathrm{Fe}] \sim$ +1.6 !). Analysis of a new high S/N spectrum by Sneden et al. (1996) yielded abundances for 20 neutron-capture elements. The abundances of 15 elements from $Z=56(\mathrm{Ba})$ to $72(\mathrm{Hf})$ match nearly perfectly a scaled solar-system r-process abundance pattern. This result, combined with the extreme overabundances of all these elements, suggests that a single prior (local) supernova explosion seeded the neutron-capture elements in the halo ISM gas cloud that condensed to become CS 22892-052. The early galactic halo should have been chemically inhomogeneous as the first wave of supernovae were occurring, and observational confirmation now exists in the exotic abundances of CS 22892-052 and in the general variations of neutron-capture element levels in UMP stars.

\section{New Approaches to the Very Heavy Element Distributions}

Thorium $(Z=90)$ is produced exclusively in the r-process and is unstable, decaying with a half-life of 14.0 Gyrs. Thus its abundance relative to lighter neutron-capture elements can in principle yield an estimate for the "age" of the galaxy. Notable attempts to analyze the Th II feature include those of Butcher (1987), Morell et al. (1992), and François et al. (1993), who determined either [Th/Nd] or [Th/Eu] ratios. CS 22892-052 has a strong Th II $4019 \AA$ absorption feature, and Sneden et al. (1996) used the Th abundance to estimate that the age of neutron-capture material gathered into CS 22892-052 is $15.2 \pm 3.7$ Gyrs. Thorium cosmochronometry is no 
simple task, and Cowan et al. (1997 and references therein) discuss the many complications. Briefly, one needs to worry about: [a] line blending problems for the Th II feature (they are severe); [b] the lack, so far, of other Th II features for analysis; [c] the choice of comparison element (the nearly pure r-process element $\mathrm{Eu}$ is a happier choice than Nd, which has mixed $\mathrm{r}$ - and s-process parentage in solar system material); [d] the production ratio of Th to the lighter neutron-capture elements (so far, a solar-system production ratio has been assumed for lack of a more reliable estimate); and [e] galactic chemical evolution effects on stable and radioactive elements.

All of these points need much work in order for thorium cosmochronometry to be reliable. Progress has been made on the production ratio of Th to other neutron-capture elements. In most metal-poor stars, one has at best measured values of [Th/Eu], and François et al. (1993) caution against over interpretation of these values. The initial $[\mathrm{Th} / \mathrm{Eu}]$ ratios generated in early galactic supernovae must be assumed, usually by analogy to solar system abundance ratios, in order to use the observed ratios in metal-poor stars to estimate the age of the galactic halo. But there is a gap of 26 elements between $\mathrm{Eu}$ and $\mathrm{Th}$ ! Even in the most favorable case of CS 22892-052, the Z gap is almost 20 between Hf and Th (the Sneden et al. 1996 Os abundance is not well determined). Therefore Cowan et al. (1996) and Sneden et al. (1998) have obtained HST high resolution UV spectra of Os $(\mathrm{Z}=76), \mathrm{Pt}(\mathrm{Z}=78)$ and $\mathrm{Pb}(\mathrm{Z}=82)$ in three bright very metal-poor giants (CS 22892-052 is too faint for this work). Their analyses demonstrate that these extremely heavy stable elements fit very well into the scaled solar-system r-process distribution of the lighter elements Ba through Eu in these stars. Thus for the r-process components of the neutron-capture elements, the supernovae that produce them seem to have a narrow range of parameters, so that approximately the same abundance distribution of these elements has been generated throughout the galaxy's history. Worries about radical departures from solar system abundance distributions in the r-process material from early galactic supernovae appear to be unfounded, and one may make more confident assumptions about initial Th production for metal-poor stars.

The neutron-capture elements from $31 \leq \mathrm{Z} \leq 55$ have been ignored here. The easily observed Sr-Y-Zr element group does not have a clean match to scaled solar system r- or s-process abundance distributions (see, eg. Cowan et al. 1995). In addition to confusions over the relative $\mathrm{r}$ - and s-process contributions to these lighter elements in metal-poor stars, Wasserburg et al. (1996) have proposed that there are two distinct r-process production sites that make the lighter and heavier neutron-capture elements (the existence of two s-process synthesis components has been known for some time). If true, it will be difficult to ever understand the nucleosynthetic 
origin(s) of these elements. To gain insight on this question, Crawford et al. (1998) have used Keck spectra of several metal-poor stars to search for other neutron-capture elements with $\mathrm{Z} \leq 55$. In Figure 2 we show their detections of one of the two neutral resonance lines of $\mathrm{Ag}(Z=47)$ and a sample match between synthetic and observed spectra for one of the stars. In this and other stars, it seems that $[\mathrm{Ag} / \mathrm{Fe}] \sim 0$, but unfortunately the most metal-poor star of the Crawford et al. sample has $[\mathrm{Fe} / \mathrm{H}] \sim-2.2$, well above the UMP star domain where the r-process dominates the neutroncapture element abundances. Clearly we need further work on this and other lighter neutron-capture element transitions in the UV spectra of more metal-poor stars. Some of these elements will be accessible to ground-based spectroscopy, but other must be attacked with HST.

We thank John Cowan, Jim Truran, Debra Burris, Caty Pilachowski, Andy McWilliam, Patrick François, and William Gacquer for helpful discussions. This work has been supported by the National Science Foundation, most recently through grant AST-9618364.

\section{References}

Beers, T. C., Preston, G. W., \& Shectman, S. A. 1992, $A J, 103,1987$

Brown, J. A., Wallerstein, G., and Zucker, D. 1997, $A J, 114,180$

Burris, D. L., Pilachowski, C. A., Armandroff, T., Sneden, C., and Cowan, J. J. 1998, in preparation

Butcher, H. R. 1987, Nature, 328, 127

Carney, B. W., Wright, J. S., Sneden, C., Laird, J. B., and Aguilar, L. A. 1997, $A J, 114$, 363

Cayrel, R. 1996, A\&A Rev., 7, 217

Cowan, J. J., Burris, D. L., Sneden, C., Preston, G. W., and McWilliam, A. 1995, ApJ, 439, L51

Cowan, J. J., Sneden, C., Truran, J. W., and Burris, D. L. 1996, ApJ, 460, L115

Cowan, J. J., McWilliam, A., Sneden, C., Burris, D. L., and Preston, G. W. 1997, $A p J$, 480,246

Crawford, J., Sneden, C., King, J. R., Deliyannis, C., and Boesgaard, A. M. 1998, in preparation

Edvardsson, B., Andersen, J., Gustafsson, B., Lambert, D. L., Nissen, P. E., and Tomkin, J. 1993, $A \mathcal{E} A, \mathbf{2 7 5}, 101$

François, P. 1996, $A \& A, \mathbf{3 1 3}, 229$

François, P., Spite, F., and Spite, M. 1993, $A \mathscr{E} A, \mathbf{2 7 4}, 821$

Fuhrmann, K., Axer, M., and Gehren, T. 1995, $A \& A$, 301, 492

Gacquer, W., and François, P. 1998, preprint

Gilroy, K. K., Sneden, C., Pilachowski, C. A., and Cowan, J. J. 1988, ApJ, 327, 298

Gratton, R. G. 1989, $A \& A$, 208, 171

Gratton, R. G., and Sneden, C. 1994, $A \mathcal{B} A, \mathbf{2 8 7}, 927$

Käppeler, F., Beer, H., \& Wisshak, K. 1989, Rep. Prog. Phys., 52, 945

Magain, P. 1989, $A \mathscr{E} A, 209,211$

Magain, P. 1995, $A \& A, 297,686$

McWilliam, A. 1997, $A R A \&$, in press

McWilliam, A., Preston, G. W., Sneden, C., \& Searle, L. 1S95, AJ, 109, 2757 

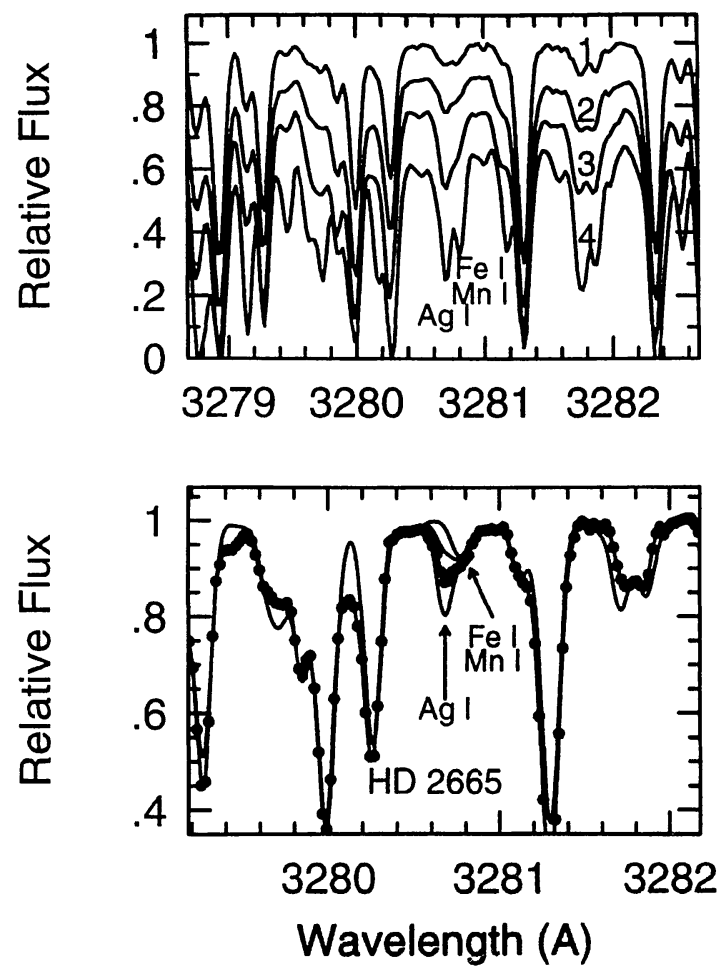

Figure 2. The detection of silver in metal-poor stars (Crawford et al. 1998). In the upper panel we show the blended Ag I line in BD +37 1458 (star 1), HD 2665 (2), HD 6755 (3), and HD 103095 (4). In the bottom panel the observed HD 2665 spectrum is given by the solid line with filled circles representing the individual spectrum points, and syntheses for this low metallicity $\operatorname{star}([\mathrm{Fe} / \mathrm{H}] \simeq-2)$ are shown for $[\mathrm{Ag} / \mathrm{Fe}]=-12.0$ (essentially no $\mathrm{Ag}$ ), $-0.5,-0.1$ (the best fit; buried under the data points), and +0.2 .

Meyer, B. S. 1994, ARA $6 A$, 32, 153

Morell, O., Källander, D., \& Butcher, H. R. 1992, $A \& A$, 259, 543

Nissen, P. E., and Schuster, W. J. 1997, $A \& A$, 326, 751

Pilachowski, C. A., Sneden, C., and Kraft, R. P. 1996, $A J, 111,1689$

Ryan, S. G., Norris, J. E., and Bessell, M. S. 1991, $A J$, 102, 303

Ryan, S. G., Norris, J. E., and Beers, T. C. 1996, $A p J, 471,254$

Sneden, C., Gratton, R. G., and Crocker, D. A. 1991, $A \& A$, 246, 354

Sneden, C., McWilliam, A., Preston, G. W., Cowan, J. J, and Burris, D. L. 1996, ApJ, 327, 298

Timmes, F. X., Woosley, S. E., and Weaver, T. A. 1995, ApJS, 98, 617

Wallerstein, G., Greenstein, J. L., Parker, R., Helfer, H. L., and Aller, L. H. 1963, ApJ, 137,280

Wasserburg, G. J., Busso, M. \& Gallino, R. 1996, ApJ, 466, L109

Woolf, V. M., Tomkin, J., and Lambert, D. L. 1995, ApJ, 453, 660

Zhao, G., and Magain, P. 1990, AEA, 238, 242 